\title{
Commentary
}

\section{Neuroethics Guiding Principles for the NIH BRAIN Initiative}

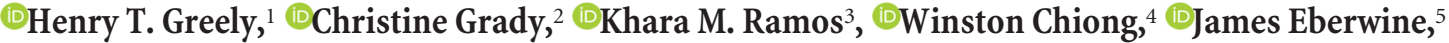

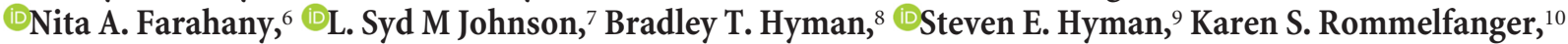 \\ and Elba E. Serrano ${ }^{11}$ \\ ${ }^{1}$ Stanford Law School, Stanford University, Stanford, California 94305, ${ }^{2}$ National Institutes of Health Clinical Center, Bethesda, Maryland 20892, ${ }^{3}$ National \\ Institute of Neurological Disorders and Stroke, National Institutes of Health, Bethesda, Maryland 20892, ${ }^{4}$ Department of Neurology, University of \\ California, San Francisco, California 94158, ${ }^{5}$ Department of Pharmacology, University of Pennsylvania, Philadelphia, Pennsylvania 19104, ${ }^{6}$ Duke University, \\ Durham, North Carolina 27708, ${ }^{7}$ Department of Humanities, Michigan Technological University, Houghton, Michigan 49931, ${ }^{8}$ Department of Neurology, \\ Massachusetts General Hospital, Harvard Medical School, Boston, Massachusetts 02129, ${ }^{9}$ Broad Institute of Massachusetts Institute of Technology and \\ Harvard University, Boston, Massachusetts 02141, ${ }^{10}$ Department of Neurology, Emory University, Atlanta, Georgia 30322, and ${ }^{11}$ Department of Biology, \\ New Mexico State University, Las Cruces, New Mexico 88003
}

\section{Introduction}

Neuroscience presents important neuroethical considerations. Human neuroscience demands focused application of the core research ethics guidelines set out in documents such as the Belmont Report. Various mechanisms, including institutional review boards (IRBs), privacy rules, and the Food and Drug Administration, regulate many aspects of neuroscience research and many articles, books, workshops, and conferences address neuroethics. (Farah, 2010; https://bioethicsarchive. georgetown.edu/pcsbi/studies.html; http:// www.neuroethicssociety.org/annualmeeting). However, responsible neuroscience research requires continual dialogue among neuroscience researchers, ethicists, philosophers, lawyers, and other stakeholders to help assess its ethical, legal, and societal implications. The Neuroethics Working Group of the National Institutes of Health (NIH) Brain Research through Advancing Innovative Neurotechnologies (BRAIN) Initiative, a group of experts providing neuroethics input to the NIH BRAIN Initiative Multi-Council Working Group, seeks to promote this dialogue by pro-

Received Aug. 13, 2018; revised 0ct. 5, 2018; accepted 0ct. 16, 2018.

We thank Dr. Walter Koroshetz and Dr. Joshua Gordon for their support of this effort and the many BRAIN Initiative-affiliated investigators and staff who provided helpful input as these principles were developed.

The authors declare no competing financial interests.

Correspondence should be addressed to: Henry T. Greely; Stanford Law

School, Stanford University, Neukom Building, Room N361, Stanford, CA 94305. E-mail: hgreely@stanford.edu.

https://doi.org/10.1523/JNEUROSCI.2077-18.2018

Copyright $\odot 2018$ the authors $\quad 0270-6474 / 18 / 3810586-03 \$ 15.00 / 0$ posing the following Neuroethics Guiding Principles (Table 1).

Two general points frame these principles. First, pursuing neuroscience research is an ethical imperative. Brain diseases and disorders cause immense suffering and have a major economic impact worldwide. Our ability to intervene medically is hampered by limited understanding of brain function and of how brain circuits go awry in disorders such as dementia, chronic pain, depression, addiction, and autism. The NIH BRAIN Initiative focuses on developing new tools and neurotechnologies to transform understanding of brain function in health and disease. That knowledge is critical to enabling novel therapies for brain disorders and thus is ethically compelled.

Second, neuroethics is vital to neuroscience research. The consideration of ethical, legal, and societal implications of neuroscience research facilitates progress in neuroscience and helps to ensure that neuroscientific advancements support human well-being. Integrating neuroethics into the NIH BRAIN Initiative serves the interests of all involved stakeholders. Success requires collaborative input from many disciplines, including neuroscience, medicine, bioethics, philosophy, law, and others.

We intend these principles to serve as points to consider for researchers, IRBs, and others involved in the conduct of BRAIN-funded research. They also may be useful to NIH leadership and advisory groups when considering future research directions (http://doi.org/
10.1523/JNEUROSCI.2091-18.2018) and likely to people and groups beyond the NIH BRAIN Initiative.

\section{Neuroethics Guiding Principles \\ 1. Make assessing safety paramount}

Human subjects protections place the highest priority on research participant safety, including physical, psychological, and emotional consequences of research participation, in the short, intermediate, and long term. This is particularly important in neuroscience research because the complexity of the human brain lends unpredictability to outcomes of intervention and may heighten the likelihood and potential severity of unexpected consequences, including those emerging at later times because of the brain's plasticity. Safety also is crucial when implementing interventions for widespread clinical use in treating brain diseases and disorders. Safety can never be guaranteed, but risks must be rigorously assessed and carefully weighed against likely benefits in both research and treatment. The development of safe interventions depends on robust experimental design throughout the research pipeline, including adherence to the highest standards for rigor and reproducibility. Early-stage research with nonhuman model systems must be carefully designed to identify potential limitations during translational phases of research. For example, new methods of neuromodulation (invasive or otherwise) may create unanticipated interactions and reverberating consequences. New geneediting technologies such as CRISPR/ 
Table 1. Neuroethics Guiding Principles

1. Make assessing safety paramount

2. Anticipate special issues related to capacity, autonomy, and agency

3. Protect the privacy and confidentiality of neural data

4. Attend to possible malign uses of neuroscience tools and neurotechnologies

5. Move neuroscience tools and neurotechnologies into medical or nonmedical uses with caution

6. Identify and address specific concerns of the public about the brain

7. Encourage public education and dialogue

8. Behave justly and share the benefits of neuroscience research and resulting technologies

CAS, while offering hope for mitigating or eliminating brain disorders, are still in their infancy and carry potential for offtarget effects. It is essential to attend to safety data from preclinical studies and to monitor safety throughout research when evaluating such innovative approaches for potential efficacy in humans. Research participants must be thoroughly informed of potential risks and benefits, as well as the possibility of unexpected safety issues.

\section{Anticipate special issues related to capacity, autonomy, and agency}

Contemporary neuroscience research may enable greater understanding of brain disorders associated with impaired, fluctuating, or diminished decision-making capacities and diminished agency (our ability to choose our actions) and autonomy (our ability to freely make informed choices). Some of these disorders may present in children, in whom these characteristics are also limited. Responsible BRAIN-funded research must study, not only "competent" and autonomous adults, but also people with diminished or developing autonomy and decision-making capacity. The challenges of a fair consent process that allows participation of those with limited, "different," or fluctuating capacity to consent are not new but require constant attention. For example, in research with patients with Alzheimer's dementia, routine assessment of how well participants receive and process information and their decision-making ability is crucial. This may prove especially challenging for patient participants with advanced forms of the disease or when research involves innovative techniques that may perturb capacity in ways unfamiliar to participants.

Some interventions may lead to unanticipated changes in preferences and agency, as in reported personality changes after deep-brain stimulation for movement disorders (Lewis et al., 2015). In contrast, patients with neuropsychiatric conditions may actively seek such alterations to enhance their agency or restore capacities. Researchers may find themselves in the paradoxical position of seeking informed consent from participants while at the same time manipulating neural processes necessary for consent capacity and autonomous choice. For example, brain stimulation paradigms may target circuits involved in reward processing and motivation. Given our limited understanding of whether excessive stimulation might undermine patient participants' future decision making, how much control regarding stimulation parameters should go to participants in alignment with their autonomy interests rather than to researchers? Researchers should be particularly cautious to preserve and monitor research participants' ability to consent, including consent to continued participation in research.

Providing participants with accurate, easy to understand, and evidence-based information about potential risks and benefits will promote well informed decisions about participation in neuroscience research (https://www.accessdata.fda.gov/ scripts/cdrh/cfdocs/cfCFR/CFRSearch. $\mathrm{cfm}$ ?fr=50.25). Care must be taken to avoid overpromising to possible participants, who may be desperate for a helpful intervention, and to discourage them from believing that personal benefits are more likely than they are.

\section{Protect the privacy and \\ confidentiality of neural data}

Research participants have a reasonable expectation of privacy regarding their neural data and that data's interpretation, which might include perceptions, emotions, memories, and thoughts. NIH BRAIN Initiative research is developing better methods to measure brain structure and activity. These data will be stored for analysis and shared often with other researchers with appropriate privacy protections to advance efforts to understand the brain. Neural data should be treated as private, sensitive information; its collection, transmission, and storage should adhere to best practices for security and encryption. Conflicts may exist between privacy/confidentiality and data sharing. For example, large, shared databases containing brain imaging data may be extremely useful for researchers studying both healthy and atypical brain functioning, but every brain is unique and someday a brain MRI might be as identifying as a fingerprint. A person with access to a shared database, as well as an individual's
MRI, might be able to determine whether the individual was in the database and, if so, obtain personal information about him or her from the "de-identified" database.

It is important that researchers and policymakers find ways to manage these problems. Research participants' confidentiality cannot be guaranteed both because of the risks of unauthorized release of identified data through hacking and the possibilities of re-identification. Research participants must be given clear information about these issues and an honest chance to decide whether to accept the risks.

\section{Attend to possible malign uses of neuroscience tools and neurotechnologies}

Novel tools and technologies, including neurotechnologies, can be used both for good ends and bad. Researchers should be mindful of possible misuses that might range from intrusive surveillance of brain states to efforts to incapacitate or impermissibly alter a person's behavior. Researchers have a responsibility to try to predict plausible misuses and ensure that foreseeable risks are understood, as appropriate, by research participants, IRBs, ethicists, and government officials. When possible, misuse should be prevented, for example, through design of the technology, such as ensuring secure wireless device connections.

\section{Move neuroscience tools and neurotechnologies into medical or nonmedical uses with caution} BRAIN Initiative research includes cutting-edge science and first-in-human applications of novel neurotechnologies. Accordingly, the likelihood of individual benefit may be low and uncertain and risks could be significant. Researchers must thoroughly identify and minimize potential research risks. A thoughtful justification of risks based on the potential benefits is essential.

Hopes for neuroscience extend beyond research into exciting prospects for novel therapeutics. In addition to safety, researchers should consider questions of efficacy and equity before novel neurotechnologies become widely available. Researchers and others involved in the NIH BRAIN Initiative should discourage the premature widespread use or inappropriate adoption of new technologies, especially those that may be offered directly to consumers or in non-health-care settings, such as in the legal system. For example, 
researchers looking for neural markers of deception or pain should be aware that segments of society may be eager to use such tools for non-health-related ends. Premature adoption of such tools before accuracy is known is not appropriate.

\section{Identify and address specific concerns of the public about the brain}

People care deeply about their minds and brains and have concerns that researchers may not sufficiently recognize. Even scientifically unjustified fears can have important consequences for public response to neuroscience work. Although sensitivity about brain-related issues varies between cultures, three examples follow.

Fear of mental invasion reaches far back into human history, as does the idea of cognitive liberty-that the freedom and privacy of one's mind (and thus brain) is sacrosanct. Some might have concerns that a beneficial improvement in ability to control the dysfunctional mind (e.g., from memory loss or seizures) also may have detrimental outcomes and potentially threatens cognitive liberty (Ienca and Andorno, 2017). Second, many people perceive their identity as being within their brain. Novel neurointerventions might disrupt that identity; for example, brain implants might alter a persons' sense of self or change their behavior in unexpected or unwelcome ways (Gilbert et al., 2017). Researchers should be aware of these justified concerns that research could "make a person someone else." Last, many consider the human mind and brain to be distinguishing, perhaps definitive, features of being human. Research with human/nonhuman brain chimeras, neural organoids, and ex vivo human brain tissue can provoke intellectual, visceral, and moral concerns, including concerns about the potential development of morally significant features in these tissues.

Researchers, funders, and others should try to identify issues arising from their research that the public might find sensitive, taking into account the possibility of sensationalized media reports. Both the public and researchers will benefit if the latter consider public concerns when planning, implementing, and discussing research, as described in the next principle.

\section{Encourage public education and dialogue}

Public trust in science is a precious commodity. To the greatest extent possible, researchers should build - and retainthat trust by keeping the public informed. Public dialogue should be bidirectional, where researchers stay abreast of the public's desires, concerns, and degree of knowledge. Some conflicts between informing the public about research as it proceeds and researchers' appropriate desires to delay sharing preliminary findings before appropriate review may be unavoidable. Nevertheless, transparency is crucial, particularly with potentially controversial research, to avoid unduly concerning the public. Being a scientist today requires not only good work, but also good communication about that work. Modern society offers scientists a wide array of ways to communicate beyond the traditional peer-reviewed paper and academic conference talk. Good ethical stewardship of one's work calls on scientists to find methods that best suit them, whether through public talks, online scholarship, creating social media content, giving interviews, or other paths. Researchers have an obligation to share knowledge both about the brain and about where we continue to be ignorant about the brain's workings, along with possible benefits and risks of research. University and government communications offices also have a critical role to play in promoting transparency.

Hyperbole is in part driven by the imaginations of scientists, the public, and neuroethicists and because hype about the next great breakthrough is widely used to hold attention. Researchers, science journalists, communications offices, and others-including neuroethicists-have essential roles to play in promoting appropriate understanding, avoiding hyperbole, and correcting overly optimistic interpretations.

\section{Behave justly and share the benefits of neuroscience research and resulting technologies}

The former Presidential Commission for the Study of Bioethical Issues wrote ". . . a fundamental principle of fairness suggests that society should seek to assure that the benefits and burdens of new technologies are shared" (https://bioethicsarchive. georgetown.edu/pcsbi/sites/default/ files/PCSBI-Synthetic-Biology-Report12.16.10_0.pdf). Early BRAIN Initiative studies are likely to be small and fairness in selection of research participants is critical because more people may want to participate than can be included given finite opportunities and participants with few options for treatment may be more open to untested options. For example, experiments testing visual prostheses may be very appealing to persons severely affected by vision loss. Similarly, the possible appeal of brainmachine interface experiments for those suffering from tetraplegia warrants careful processes for selecting early trial participants.

As technologies are found to be safe and effective and enter clinical use, attention to widespread sharing of the benefits of those technologies and interventions will become a priority. Limited access to safe and effective neural technologies should not exacerbate existing health disparities or inequalities, but neither should the burdens of research fall disproportionately on those who lack access to established interventions.

\section{Conclusion}

We offer these principles as points to consider to help researchers and other stakeholders better navigate the difficult questions that the NIH BRAIN Initiative research will pose to society. Neuroscience research holds great promise. With that promise comes great responsibility for diverse stakeholders to ensure both that the promise can be fulfilled and that it is not distorted. Neuroethics can help to achieve this goal through published discussions, research, consultation on complex ethical issues arising from research, the application of principles like those herein.

\section{References}

Farah MJ (2010) Neuroethics: an introduction with readings. Cambridge, MA: MIT.

Gilbert F, Goddard E, Viaña JNM, Carter A, Horne M (2017) I miss being me: phenomenological effects of deep brain stimulation. AJOB Neuroscience 8:96-109. CrossRef

Ienca M, Andorno R (2017) Towards new human rights in the age of neuroscience and neurotechnology. Life Sci Soc Policy 13:5. CrossRef Medline

Lewis CJ, Maier F, Horstkötter N, Zywczok A, Witt K, Eggers C, Meyer TD, Dembek TA, Maarouf M, Moro E, Zurowski M, Woopen C, Kuhn J, Timmermann L (2015) Subjectively perceived personality and mood changes associated with subthalamic stimulation in patients with Parkinson's disease. Psychol Med 45:73-85. CrossRef Medline 